0.001); 1 patient had to undergo a medical examination versus 3 from the controls $(p=0.96)$; and for 2 patients restrictions in the contract were imposed versus 0 from the controls $(\mathrm{p}=$ $0.005)$.

19 out of 38 patients effected an additional work-disability insurance after the diagnosis of AS. Six of them were obliged to supply medical information versus 0 from the control group (n $=19)(\mathrm{p}=0.027) ; 2$ patients had to undergo a medical examination versus 0 from the controls $(p=0.47$ ); for 2 patients restrictions in the contract were imposed versus 0 from the controls $(\mathrm{p}=0.47)$.

25 out of 57 patients effected a life insurance after the diagnosis of AS. Seventeen patients were obliged to supply medical information versus 2 from the control group $(\mathrm{n}=32)$ ( $\mathrm{p}<$ 0.001); 6 patients had to undergo a medical examination versus 3 from the controls $(p=0.26)$; for 7 patients restrictions in the contract were imposed versus 1 from the controls $(p=0.02)$.

Conclusion In The Netherlands, AS patients encounter substantial hindrances in effecting insurances. Especially in effecting a health or life insurance medical information is often requested, which may lead to restrictions in the insurance contract.

\section{SAT0232 MANAGEMENT OF CHRONIC MUSCULOSKELETAL PAIN}

M Pileckyte, N Misiuniene. Department Rheumatology, Kaunas Medical University, Kaunas, Lithuania

\subsection{6/annrheumdis-2001.868}

\section{Background}

Objectives Of the study were to explore the management of sufferers with chronic musculoskeletal pain (CMP) syndromes.

Methods A random sample of subjects was selected in the vicinity of two main outpatient clinics of the city Kaunas. All subjects aged 25-74 were requested to indicate on a mannequin any pain which had been lasting at least $24 \mathrm{~h}$ since the last month. Subjects who met the ACR 1990 definition for chronic widespread pain (CWP) and subjects who satisfied the standard definition of chronic low back pain (CBP) but not the ACR definition for CWP were interviewed according to a standard questionnaire. A follow-up screening of all the subjects was carried out after 6 months. Subjects who still reported CWP and CBP were classified as "persistent" CWP and CBP sufferers. Outpatient medical records of all these subjects were analysed.

Results The study population comprised 1,404 subjects, 1,030 being aged $25-74$. The response rate was $83.01 \%$. The average age of respondents was $49.29 \pm 13.09,61.64 \%$ being females. The prevalence of CWP was $18.60 \%$ and CBP- $12.28 \%$. After 6 months $88.68 \%$ of subjects with CWP and $72.38 \%$ with CBP participated in the follow-up screening. "Persistent" CWP was present in $79.43 \%$ and "persistent" CBP- in $47.37 \%$ of respondents. The majority of subjects with CWP (98.08\%) and CBP $(88.89 \%)$ consulted the general practitioner (GP) during the last year, mainly because of CMP. Many CWP (54.90\%) and CBP $(37.50 \%)$ sufferers consulted the GP 6-10 times during the last year. They were ordered a lot of investigations by the GP and were referred to specialists for medical advice (e.g., to a neurologist- $56.86 \%$ of CWP and $66.67 \%$ of CBP sufferers but to a rheumatologist- only $3.92 \%$ of CWP and $16.67 \%$ of CBP sufferers). The diagnosis was altered only in a very few cases. CBP was poorly diagnosed and fibromyalgia syndrome was not diagnosed at all by the GP. CMP patients were treated mainly with nonsteroidal anti-inflammatory drugs. No one received antidepressants, only $29.41 \%$ of CWP and $54.17 \%$ of CBP sufferers were prescribed rehabilitation procedures, but often they were hospitalised (27.45\% of CWP and $29.17 \%$ of CBP cases) during the last year. Quite a big number of CWP sufferers experienced either short-time or permanent disability.

Conclusion CMP syndromes are common in the outpatient population. More attention and priority for education of medical professionals concerning early diagnosis and appropriate treatment of CMP should be given by responsible health care authorities.

\section{SAT0233 A RANDOMISED COMPARISON OF CARE PROVIDED BY A CLINICAL NURSE SPECIALIST, INPATIENT TEAM CARE AND DAY PATIENT TEAM CARE IN RHEUMATOID ARTHRITIS (RA)}

${ }^{1} \mathrm{GJ}$ Tijhuis, ${ }^{2} \mathrm{AH}$ Zwinderman, ${ }^{1} \mathrm{JM}$ Hazes, ${ }^{3} \mathrm{WB}$ Van den Hout, ${ }^{1} \mathrm{FC}$ Breedveld, ${ }^{1} \mathrm{TP}$ Vliet Vlieland. ${ }^{1}$ Rheumatology; ${ }^{2}$ Medical Statistics; ${ }^{3}$ Medial Decision Making, Leiden University Medical Centre, Leiden, Netherlands

10.1136/annrheumdis-2001.869

Background The role of clinical nurse specialists in the management of patients with RA is evolving, and their effectiveness in comparison with care provided by a rheumatologist alone has been established. However, controlled studies showing how the effectiveness of clinical nurse specialists compares to that of multi-disciplinary team care, a treatment strategy that is considered to be optimal in chronic disease management, are lacking. Objectives The first aim of the study was to compare the clinical effectiveness of care delivered by a clinical nurse specialist with inpatient team care and day patient team care in patients with RA. The second aim was to determine factors predicting clinical response.

Methods A multi-centre, randomised controlled clinical trial including consecutive outpatients with RA and progressive functional limitations over the past 6 weeks. Clinical assessments recorded on study entry, weeks $6,12,26$ and 52 comprised the HAQ, MACTAR functional index, RAND-36 quality of life questionnaire, health utility rating scale (RS) and DAS. Patients? satisfaction with care was measured on a visual analogue scale. Within-group differences between baseline and follow-up scores were tested with the Wilcoxon signed rank matched-pairs test. Co-variance analysis was used to analyse between-group differences and to determine factors predicting clinical response.

Results 210 patients (median age 58 years, median disease duration 1.8 years and median HAQ score 1.25 ) were included in the study. Within all 3 groups functional status, quality of life, health utility and disease activity improved significantly over time (all P-values <0.05). A comparison of clinical outcome among the three groups and a comparison between the nurse specialist group and the inpatient and day patient care groups together did not show any sustained significant differences. Subgroup analysis showed that with increasing age, the most favourable outcome according to the HAQ shifted from care provided by a clinical nurse specialist and inpatient care to day patient care. Patients? satisfaction with care was significantly lower in the nurse specialist group than in the inpatient and day patient care groups $(\mathrm{P}<0.001)$.

Conclusion Care provided by a clinical nurse specialist appears to have a similar clinical outcome in comparison with inpatient and day patient team care in patients with RA. Age was the only factor related to differences in functional outcome between the three treatment groups. The choice of management strategy may, 
apart from age, further be dependent on the availability of facilities, the preferences of patients and health care providers and on economic considerations.

\section{SAT0234 EVOLUTION IN THE USE OF NSAIDS BETWEEN 1991 AND 2000. IMPACT OF THE NEW MARKETED DRUGS}

${ }^{1} \mathrm{C}$ Llop, ${ }^{2} \mathrm{~S}$ Paredes. ${ }^{1}$ Pharmacy Unit, Catalonian Health Service, Tarragona; ${ }^{2}$ Rheumatology Service, Assistencial Service, Amposta, Spain

10.1136/annrheumdis-2001.870

Background Non-steroidal anti-inflammatory drugs (NSAIDs) are widely used drugs. In Catalonia (Spain), this group of drugs is the fourth most used group in the number of packages dispensed in recent years. Furthermore, new NSAIDs have recently become available on the Spanish pharmaceutical market.

Objectives To study the evolution in the use of NSAIDs in the province of Tarragona (Catalonia, Spain), with 450,000 inhabitants, during the period between 1991 and 2000. We also studied the commercial impact of the new marketed drugs in recent years.

Methods Data of all NSAIDs prescribed and dispensed in the Public Health System during the period of the study were obtained from the Catalonian Pharmacy Database. These data were converted into Defined Daily Doses (DDD) per 1,000 inhabitants per day (DHD), according the definition of the Nordisk Council of Medicine. Population of the province was obtained from the official census of various years.

Results The total amount of NSAIDs used has increased in these ten years from 24.12 to 42.54 DHD. Throughout the ten years period diclofenac, piroxicam, naproxen, ibuprofen, aceclofenac and indomethacin were the most used NSAIDs. The use of ibuprofen increased greatly and in the last year was the second most used drug in the group. Diclofenac and naproxen were used a lot throughout the period and even increased in the last years. Piroxicam and indomethacin decreased slightly over the period studied.

The use of new NSAIDs (dexketoprofen, nimesulide, celecoxib, lornoxicam and particularly meloxicam and rofecoxib) increased considerably. Rofecoxib was the fourth most used drug in the group in its first year on the market. New NSAIDs are more than 20\% of the total NSAIDs used in the year 2000. Since the new NSAIDs have become available, other minoritary drugs such as ketoprofen, flurbiprofen or tenoxicam have been used much less.

Conclusion Despite the considerable number of NSAIDs available in Spain, only ten represent almost 95\% of the total NSAIDs used in the year 2000. Concerns about a possible overprescription of these drugs are reflected in the increase of use between 1991 and 2000. Data from the last year show that more than 40 people out of every 1,000 daily use a NSAID. The increase in the use of ibuprofen is noticeable but the new NSAIDs have had a commercial impact that was both considerable and fast. We conclude that aggressive advertising by pharmaceutical companies greatly influences the use and the selection of these drugs.

\section{REFERENCES}

1 Brooks PM, Day RO. Non-steroidal anti-inflammatory drugs. Differences and similarities. N Engl J Med. 1991;324:1716-25

2 Llop C, Paredes S, Llor C. Criteria for selecting and using non-steroidal antiinflammatory drugs in primary health care. Fam Practice 2000;17:63-5

\section{SAT0235 DETERMINANTS OF PRODUCTIVITY COSTS IN AS. COMPARATIVE STUDY IN THE NETHERLANDS, FRANCE AND BELGIUM}

${ }^{1} \mathrm{AH}$ Boonen, ${ }^{1} \mathrm{D}$ Van der Heijde, ${ }^{2} \mathrm{H}$ Schouten, ${ }^{1} \mathrm{R}$ Landewe, ${ }^{3} \mathrm{~F}$ Guillemin, ${ }^{4} \mathrm{M}$ Dougados, ${ }^{5} \mathrm{~K}$ De Vlam, ${ }^{5} \mathrm{H}$ Mielants, ${ }^{1} \mathrm{~A}$ Spoorenberg, ${ }^{1} \mathrm{~S}$ Van der Linden. ${ }^{1}$ Rheumatology; ${ }^{2}$ Statistics, University Hospital Maastricht, Maastricht, The Netherlands; ${ }^{3}$ Rheumatology, Ecole Sante Publique, Nancy; ${ }^{4}$ Rheumatology, Hopital Cochin, Paris, France; ${ }^{5}$ Rheumatology, University Hospital Gent, Gent, BelgiumBackground

10.1136/annrheumdis-2001.871

Objectives To evaluate determinants of productivity costs in ankylosing spondylitis (AS) in three European countries.

Methods 216 consecutive prevalent AS out-patients started a 2 year longitudinal cost-of-illness study. AS-related health resource utilisation was collected by questionnaires every 2 months. Productivity costs were calculated by friction cost (FC) method and human capital (HC) approach. Cox proportional hazard analysis with costs as dependent variable was used to assess if ?country? was a determinant of productivity costs after controlling for age, gender, disease duration and time integrated disease activity (BASDAI:0-10; higher values indicating higher disease activity) if FC were the outcome or baseline physical function (BASFI:010; higher values indicating worse function) if $\mathrm{HC}$ costs were the outcome.

Results 209 patients completed follow-up. In the Netherlands $(\mathrm{NL}),(\mathrm{F})$ and (B) respectively, 70, 69 and 74\% of patients were male, mean age 46 (sd:12), 38 (sd: 12) and 42 (sd: 14) yr, mean disease duration 24 (sd:12), 14 (sd:9) and 13 (sd:10) yr and 34, 13 and 24\% had manual professions. 196 patients were in working age and 113 patients had a paid job.

\begin{tabular}{lll} 
Abstract SAT0235 & Table 1 & \\
\hline & Friction Costs $(\mathbf{n}=113)$ & Human Capital Costs $(\mathrm{n}=196)$ \\
& HR $[95 \% \mathrm{Cl}](\mathbf{p})$ & HR $[95 \% \mathrm{Cl}](\mathbf{p})$ \\
\hline Gender (female) & $\mathrm{ns}$ & $\mathrm{ns}$ \\
Age (HR/yr) & $1.08[1.04-1.2](0.0001)$ & $1.04[1.02-1.06](0.0001)$ \\
Disease duration & $\mathrm{ns}$ & $\mathrm{ns}$ \\
BASDAl (HR/point) & $1.2[1.1-1.3](0.04)$ & Not included as variable \\
BASFI (HR/point) & Not included as variable & $1.2[1.1-1.3](0.0001)$ \\
Manual profession & $\mathrm{ns}$ & $\mathrm{ns}$ \\
Country NL & $2.4[1.3-4.5](0.007)$ & $2.8[1.2-4.7](0.0007)$ \\
\hline
\end{tabular}

Conclusion In AS, productivity costs determined by FC and HC approach are significantly associated with the country studied. This finding has important consequences for the generalizability of health economic studies.

\section{SAT0236 A LONGITUDINAL EVALUATION OF THE EFFECT OF A DECISION-SUPPORT SYSTEM ON OUTCOME IN RHEUMATOID ARTHRITIS}

J Fransen, G Stucki, T Langenegger, M Seitz, BA Michel. SCQM C/O. Department of Rheumatology, University Hospital, Zurich, Switzerland

\subsection{6/annrheumdis-2001.872}

Background Management of patients with RA is challenging and poses specific problems. With the help of a decision-support system (Swiss Clinical Quality Management in RA) that gives the rheumatologist feedback on treatment outcomes, the treatment 